\title{
Estudo experimental em cães da ação protetora de solução cardioplégica de lidocaína e potássio
}

\author{
Altamiro Ribeiro DIAS*, Paulo Sampaio GUTIERREZ*, Maria de Lourdes HIGUCHI*, \\ Rodrigo LOURENÇÃO*, Everaldo MIRANDA*, Benedito dos SANTOS*, Maria Cristina Donadio \\ ABDUCH*, Ricardo Ribeiro DIAS*, Noedir A. G. STOLF*, Sergio Almeida de OLIVEIRA*
}

RBCCV 44205-575

Dias A R, Gutierrez P S, Higuchi M L, Lourenção R, Miranda E, Santos B, Abduch M C D, Dias R $R$, Stolf N A G, Oliveira S A - Estudo experimental em cães da ação protetora de solução cardioplégica de lidocaína e potássio. Rev Bras Cir Cardiovasc 2002; 17(1): 79-89.

RESUMO: Objetivo: Os autores estudam experimentalmente em cães a ação cardioplégica de solução sangüínea normotérmica contendo lidocaína e potássio.

Material e Método: Foram operados 14 animais, de cerca de $25 \mathrm{~kg}$, sob anestesia geral e intubação traqueal. Os cães foram submetidos a esternotomia mediana, sendo canulados a aorta ascendente e o átrio direito, para colocação em perfusão extracorpórea. Durante o procedimento, foram feitas dosagens gasométricas e de eletrólitos, visando à manutenção de boas condições cardiocirculatórias. Em 5 cães, os quais compõem grupo designado não protegido (NP), foi feita a parada cardíaca anóxica, através de pinçamento da raiz da aorta, durante 120 minutos. Nesses animais não foi tomada nenhuma medida visando à proteção miocárdica. No outro grupo, designado grupo protegido $(\mathrm{P})$, o procedimento foi semelhante, sendo que, após o pinçamento da aorta, foi feita proteção cardioplégica. A técnica de cardioplegia consistiu em indução com injeção de $100 \mathrm{mg}$ de lidocaína e 2,5 mEq de potássio, diluídos em $60 \mathrm{ml}$ de sangue da linha arterial, após o que infundiu-se sangue durante 30 segundos. A parada cardíaca foi imediata. A fase de manutenção consistiu em infusões de sangue da linha arterial, por 30 segundos, de $20 \mathrm{em}$ 20 minutos.

Resultados: Todos os animais desse grupo protegido sobreviveram enquanto que no grupo não protegido, após o restabelecimento da circulação coronariana pelo desclampeamento aórtico, todos vieram a falecer. Em todos os 14 animais foram feitas determinações ecocardiográficas de fração de ejeção e delta $D$, sendo esses valores normais. Verificamos que, no grupo protegido, houve queda desses valores de cerca de $30 \%$ na primeira hora pós suspensão da perfusão. Esses valores foram determinados novamente cerca de 5 horas pós suspensão da perfusão e revelaram-se normais. Após essa segunda avaliação ecocardiográfica, os animais foram sacrificados para estudo anatomopatológico à microscopia óptica e eletrônica. Estes estudos revelaram intensas alterações morfológicas compatíveis com necrose celular no grupo não protegido. No grupo de animais em que se utilizou a cardioplegia, as alterações microscópicas e utramicroscópicas foram inexpressivas.

Conclusão: A técnica de cardioplegia apresentada garantiu a sobrevida dos animais, após 120 minutos de parada cardíaca anóxica, com boa performance ecocardiográfica e morfológica, dentro da metodologia utilizada.

DESCRITORES: Soluções cardioplégicas. Lidocaína, uso terapêutico. Potássio, uso terapêutico.

\footnotetext{
Trabalho realizado na Divisão de Cirurgia do Instituto do Coração do Hospital das Clínicas da Faculdade de Medicina da Universidade de São Paulo, em conjunto com Divisão de Experimentação, Divisão de Diagnóstico por Imagem e Laboratório de Anatomia Patológica do InCor -HC.FMUSP. São Paulo, SP, Brasil.

Trabalho recebido para publicação em janeiro de 2001.

*Do Instituto do Coração do Hospital das Clínicas da Faculdade de Medicina da Universidade de São Paulo.

Endereço para correspondência: Altamiro Ribeiro Dias. Av. Dr. Enéas de Carvalho Aguiar, 44, Cerqueira César, São Paulo, SP, Brasil CEP 05403 - 000 Tel. (11) 3069-5234 / (11) 3069-5432. Fax: (11) 3069-5414. e-mail: altamiro_rd@incor.usp.br
} 
Dias A R, Gutierrez P S, Higuchi M L, Lourenção R, Miranda E, Santos B, Abduch M C D, Dias R R, Stolf N A G, Oliveira S A Estudo experimental em cães da ação protetora de solução cardioplégica de lidocaína e potássio. Rev Bras Cir Cardiovasc 2002; 17(1): 79-89.

\section{INTRODUÇÃO}

A lidocaína tem sido usada como agente antiarrítmico rotineiramente ${ }^{(1)}$, havendo na literatura vários trabalhos onde esse emprego é analisado.

Estudos farmacológicos de BIGGER e HOFFMAN $^{(2)}$ demonstraram que drogas antiarrítmicas do grupo da lidocaína produzem apenas pequenas alterações na despolarização e velocidade de condução nas fibras miocárdicas de Purkinge, quando os valores do potencial de repouso transmembrana são normais. Entretanto, os efeitos depressores sobre esses parâmetros são muito intensificados quando a membrana é despolarizada ou quando a freqüência de excitação está aumentada. A lidocaína acelera a repolarização da membrana.

Em seres humanos, para atingir concentrações eficazes rapidamente, a droga é infundida endovenosamente na dosagem de 0,7 a $1,4 \mathrm{mg} / \mathrm{kg}$ de peso corporal, podendo ser necessária uma segunda dose em 5 minutos. Não se deve infundir mais que 200 a $300 \mathrm{mg}$ em uma hora.

O metabolismo da droga é hepático e sua meia vida é de 100 minutos ${ }^{(3)}$.

Demonstra-se que animais submetidos experimentalmente à isquemia miocárdica por ligaduras transitórias de ramos coronarianos, quando protegidos por infusões de soluções com lidocaína, praticamente não apresentam arritmias quando reperfundidos, bem como as alterações histoquímicas são inexpressivas, sendo também melhor o desempenho do ventrículo esquerdo(4-13).

LEICHER et al.(13), em estudo experimental relatam as ações da lidocaína usada em cardioplegia sangüínea. Demonstram que a licocaína:

- Induz a parada cardíaca pela inibição do influxo de sódio e da despolarização da membrana celular;

- Tem propriedades antiarrítmicas que reduzem a incidência de arritmias no período de reperfusão;

- Inibe o influxo celular de cálcio, bem como a liberação do cálcio intracelular;

- Tem ação inibidora sobre a atividade enzimática da fosfolipase A, o que leva a um efeito estabilizador da membrana celular.

O uso da lidocaína em soluções cardioplégicas, isoladamente e/ou associada a eletrólitos como potássio, magnésio e outros componentes, tem sido freqüente. Vários trabalhos(14-18) relatam bom desempenho ventricular e menor incidência de arritmias, quando se emprega a lidocaína como agente cardioplégico.
DEMY et al.(19) fazem uma análise crítica do emprego de diferentes soluções cardioplégicas e do empirismo, que, no entender dos autores, cerca o assunto. Realçam o fato de que várias soluções cardioplégicas não foram adequadamente testadas e vêm sendo usadas clinicamente sem sólida base experimental.

O objetivo deste trabalho é avaliar experimentalmente em cães, uma nova proposta de cardioplegia. Esta proposta consiste no emprego de soluções sangüíneas normotérmicas e pode ser dividida em 2 fases: a 1 a fase é chamada de indução, a qual é feita através da injeção de solução sangüínea com lidocaína associada a pequena concentração de potássio, imediatamente após o pinçamento da aorta.

A 2 ${ }^{\text {a }}$ fase, chamada de manutenção, é obtida através da injeção de sangue da linha arterial, durante 60 segundos, sob pressão da linha arterial, repetidas a cada 20 minutos.

Tomou-se como tempo de estudo o intervalo de 120 minutos.

Usou-se, como comparação, animais colocados em perfusão, com a mesma técnica, submetidos a parada anóxica, sem nenhuma proteção.

É preciso frisar que, no presente trabalho, não se pretendeu comparar esta técnica de cardioplegia com nenhuma outra, e, sim, tão somente com ausência de proteção miocárdica.

Pelas suas características, convencionou-se chamar de parada cardíaca aeróbica a esta técnica de cardioplegia.

\section{MATERIAL E MÉTODO}

Foram estudados 2 grupos de cães, com peso variando entre 20 e $25 \mathrm{~kg}$. Todos animais foram submetidos a anestesia geral e ventilação por intubação endotraqueal, após o que foram operados mediante esternotomia mediana, sendo colocados em perfusão extracorpórea, canulando-se a aorta ascendente, próximo ao tronco braquiocefálico e o átrio direito. Procurou-se manter boas condições hemodinâmicas, bem como parâmetros hidroeletrolíticos, gasométricos e diurese normais. Em todos os animais foi utilizado o oxigenador de membranas pediátrico, os quais foram cedidos gentilmente pela Braile Biomédica.

O primeiro grupo, designado não protegido (NP), é composto de 5 cães, os quais após a instalação e estabilização da perfusão extracorpórea, foram submetidos à parada cardíaca anóxica através do 
Dias A R, Gutierrez P S, Higuchi M L, Lourenção R, Miranda E, Santos B, Abduch M C D, Dias R R, Stolf N A G, Oliveira S A Estudo experimental em cães da ação protetora de solução cardioplégica de lidocaína e potássio. Rev Bras Cir Cardiovasc 2002; 17(1): 79-89.

pinçamento transversal da aorta ascendente, imediatamente antes da sutura em bolsa através da qual foi introduzida a cânula aórtica. Entre o plano valvar e a pinça, foi introduzido um cateter para a aspiração durante o pinçamento. O tempo total de anóxia foi de 120 minutos ininterruptos, não tendo sido tomada nenhuma medida para a proteção miocárdica. Todos os animais não se recuperaram, quando da reperfusão miocárdica após 120 minutos de pinçamento aórtico.

O segundo grupo, designado protegido $(P)$, é composto de 9 cães e foi operado analogamente. Nesse grupo, entretanto, foi utilizada a técnica de cardioplegia apresentada por MIRANDA et al. ${ }^{(20)}$, a qual consiste numa fase dita de indução, onde se produz a parada cardíaca, e na chamada fase de manutenção, onde o coração permanece parado durante o tempo de pinçamento da aorta.

A chamada indução é obtida pela injeção de 100 mg de lidocaína e 2,5 mEq de potássio, diluídos em $60 \mathrm{ml}$ de sangue da linha arterial, sendo essa injeção feita através de uma derivação da linha arterial. Imediatamente após a injeção em bolo destes 60 $\mathrm{ml}$, injeta-se sangue da linha arterial durante 30 segundos. A parada cardíaca é imediata.

A fase de manutenção consiste na injeção repetida de sangue da linha arterial, durante 30 segundos, de 20 em 20 minutos, ao longo de todo o período de pinçamento aórtico. Todos os 9 cães se recuperaram ao término do período de anóxia.

Todos os cães foram submetidos a estudos da função ventricular com a utilização de ecocardiogramas transepicárdicos com aparelho Aloka SSD 870 e transdutor de $5,0 \mathrm{mHZ}$. Os exames foram gravados em vídeo Panasonic AG-6200.

Os estudos ecocardiográficos foram realizados em 3 tempos: 1ํ basal, imediatamente antes do início da perfusão. O $2^{\circ}$ exame foi feito, uma hora após a suspensão de perfusão, nos cães sobreviventes (Grupo P). O 3ำ exame foi feito cerca de 5 horas após a suspensão da perfusão. Através dessas avaliações mediu-se a função sistólica (Delta D) e a fração de ejeção (FE). Em cães normais os valores de Delta D variam de 30 a $45 \%$ e os da FE são iguais ou maiores que 0,65 (21-23).

Ao término da experimentação, os corações dos 14 animais foram removidos para estudos anatomopatológicos sob microscopia óptica e eletrônica.

Para microscopia óptica foram retirados fragmentos das paredes anterior e diafragmática do ventrículo esquerdo, bem como um fragmento da parede anterior do ventrículo direito. Após processamento habitual, os cortes foram emblocados em parafina. Foram produzidas secções de 3 a 4 micrômetros, as quais foram coradas pela hematoxilina e eosina.

A observação microscópica histológica, sob aumento de 400 vezes, abordou os seguintes aspectos no miocárdio: 1) edema intersticial; 2) infiltração de neutrófilos; 3) infiltração de mononucleares; 4) congestão, hemorragia, bandas de contração e necrose com presença de fibras homogêneas.

Todas essas variáveis foram quantificadas em cruzes, variando de 0 a 3 cruzes, de acordo com a seguinte padronização:

1) Edema intersticial

Esta avaliação foi subjetiva, de tal forma que as cruzes variaram com a maior ou menor separação das fibras miocárdicas.

2) Infiltração de neutrófilos

0 Ausência de infiltração

+ Presença de 5 ou mais neutrófilos em até $20 \%$ dos campos de 400 aumentos ou de menos que 5 destas células em mais de 207 dos campos.

++ Presença de 5 ou mais neutrófilos em 20 a $50 \%$ dos campos de $400 x$

+++ Presença de 5 ou mais neutrófilos em $50 \%$ ou mais dos campos de $400 x$

3) Infiltração de mononucleares

0 Ausência de infiltração

+ Presença de 10 ou mais mononucleares em até $20 \%$ dos campos de $400 x$ ou de menos que 10 destas células em mais de $20 \%$ dos campos

++ Presença de 10 ou mais mononucleares em 20 a $50 \%$ dos campos de $400 x$

+++ Presença de 10 ou mais mononucleares em $50 \%$ ou mais dos campos de $400 x$.

4) Congestão, hemorragia, bandas de contração e necrose com presença de fibras homogêneas.

0 Ausência de lesão

+ Presença da lesão em até $10 \%$ dos campos histológicos

++ Presença da lesão em 10 a $25 \%$ dos campos histológicos

+++ Presença da lesão em mais de $25 \%$ dos campos histológicos

Foi elaborado um escore para cada variável em cada animal, que corresponde à soma ponderada dos valores de cada lâmina examinada, atribuindo-se peso 2 a cada um dos cortes do ventrículo 
Dias A R, Gutierrez P S, Higuchi M L, Lourenção R, Miranda E, Santos B, Abduch M C D, Dias R R, Stolf N A G, Oliveira S A Estudo experimental em cães da ação protetora de solução cardioplégica de lidocaína e potássio. Rev Bras Cir Cardiovasc 2002; 17(1): 79-89.

esquerdo e peso 1 ao ventrículo direito. Dessa forma, o ventrículo esquerdo tem um peso de 4 para 1 em relação ao ventrículo direito, contemplando-se, assim, sua maior massa muscular. A análise e a quantificação foram feitas sem que 0 avaliador tivesse conhecimento do grupo a que cada cão pertencia.

É preciso assinalar que, das alterações acima descritas, tem particular importância a necrose de fibras miocárdicas em banda de contração. Não foram feitos cortes seriados.

Para microscopia eletrônica, os fragmentos após recortes adequados, foram fixados em glutaraldeído a $1 \%$, sendo pós-fixados em tetróxido de ósmio e, após os procedimentos habituais, foram embebidos em araldite. Seções de 60 nanômetros foram examinadas em ultramiocroscopia Philips 301. Não foram feitos estudos planimétricos.

Foi realizada análise subjetiva dos fragmentos submetidos a exame ao microscópio eletrônico. Tais fragmentos foram retirados de diversas porções dos 2 ventrículos e seu número variou de 2 a 7 . Os aumentos finais utilizados na observação situaramse entre 3850 a 112000 vezes.

Avaliou-se a presença das seguintes características ultraestruturais nas células miocárdicas: interrupção do sarcolema, dilatação do retículo sarcoplasmático, espaços claros no sarcoplasma, aumento de lípides, diminuição do glicogênio, alterações mitocondriais tais como: tumefação, cristólise e presença de densidades amorfas. Foi avaliado, também, a banda I alargada, desaparecimento focal de miofilamento, bandas de contração e condensação de cromatina na periferia dos núcleos.
Foram vistas alterações menores em todos os casos, tais como dilatação do retículo sarcoplasmático, espaços claros no sarcoplasma, aumento de lípides, diminuição do glicogênio, alterações mitocondriais tais como: tumefação, cristólise e presença de densidades amorfas. Foi avaliado, também, a banda alargada, desaparecimento focal de miofilamentos, bandas de contração e condensação de cromatina na periferia dos núcleos.

Foram vistas alterações menores em todos os casos, tais como dilatação do retículo sarcoplasmático, certo grau de tumefação mitocondrial bem como retificação e destruição das cristas desta organela.

A quantidade de lípides e, mais ainda de glicogênio foi pouco valorizada por sua avaliação ser muito subjetiva.

De acordo com o exposto, os casos foram considerados como de alterações leves (L), moderados (M) e graves (G) (Tabela 1).

Registre-se que essa classificação tomou em consideração apenas a existência ou não das alterações, sem quantificá-las. Assim sendo, casos com alterações mais difusas podem estar igualados a outros em que as alterações eram mais focais.

Para facilitar a exposição, convencionou-se reunir valores próximos de escores, agrupando-se resultados relativamente semelhantes; assim é que quando a variação foi de 1 a 5 , a alteração foi designada como leve (L).

Quando a variação for de 6 a 9, a alteração será designada como moderada (M).

TABELA 1

\begin{tabular}{|c|c|c|c|c|c|c|c|c|c|c|c|c|c|c|}
\hline \multicolumn{15}{|c|}{ ALTERAÇÕES ENCONTRADAS À ULTRAMICROSCOPIA } \\
\hline $\begin{array}{l}\text { Proteção } \\
\text { miocárdia }\end{array}$ & NP & $\mathbf{P}$ & $\mathbf{P}$ & NP & $\mathbf{P}$ & NP & NP & NP & $\mathbf{P}$ & $\mathbf{P}$ & $\mathbf{P}$ & $\mathbf{P}$ & $\mathbf{P}$ & $\mathbf{P}$ \\
\hline Cão & 1 & 2 & 3 & 4 & 5 & 6 & 7 & 8 & 9 & 10 & 11 & 12 & 13 & 14 \\
\hline $\begin{array}{l}\text { Densidasde amorfas } \\
\text { mitocondriais }\end{array}$ & S & & $\mathrm{S}$ & S & & S & $S$ & $\mathrm{~S}$ & & & & & & $S$ \\
\hline $\begin{array}{l}\text { Espaços claros } \\
\text { sarcoplasmáticos }\end{array}$ & $S$ & $S$ & $S$ & & & & & $S$ & & & & $S$ & $S$ & \\
\hline $\begin{array}{l}\text { Banda I muito } \\
\text { evidente }\end{array}$ & & $S$ & $\mathrm{~S}$ & & & & $S$ & & $S$ & $S$ & & $\mathrm{~S}$ & & S \\
\hline $\begin{array}{l}\text { Desaparecimento } \\
\text { focal de } \\
\text { miofilamentos }\end{array}$ & $S$ & & $S$ & & & & $S$ & $S$ & & $S$ & $S$ & & & \\
\hline $\begin{array}{l}\text { Bandas de } \\
\text { contração }\end{array}$ & S & & $\mathrm{S}$ & & & & $S$ & & & & & & & \\
\hline $\begin{array}{l}\text { Condensação de } \\
\text { cromatina na } \\
\text { periferia do núcleo }\end{array}$ & $S$ & & $S$ & & & & S & S & & & & & & \\
\hline Avaliação Geral & $M$ & $\mathrm{~L}$ & G & $\mathrm{G}$ & $\mathrm{L}$ & $\mathrm{M}$ & $\mathrm{G}$ & $\mathrm{G}$ & $\mathrm{L}$ & $M$ & $M$ & $\mathrm{~L}$ & $M$ & G \\
\hline
\end{tabular}

À esquerda observam-se as variáveis analisadas à ultramicroscopia e à direita os resultados obtidos nos diferentes animais. $\mathrm{S}=$ Presença da alteração em questão; $L$ = Leve alteração; $M$ = Moderada alteração; $G$ = Grave alteração. 
Dias A R, Gutierrez P S, Higuchi M L, Lourenção R, Miranda E, Santos B, Abduch M C D, Dias R R, Stolf N A G, Oliveira S A Estudo experimental em cães da ação protetora de solução cardioplégica de lidocaína e potássio. Rev Bras Cir Cardiovasc 2002; 17(1): 79-89.

Quando o escore for igual ou superior a 10, a alteração será designada como severa.

Não havendo alterações, a representação será A - ausente.

É preciso assinalar que, das alterações acima descritas, tem particular importância a necrose de fibras miocárdicas em banda de contração.

A análise estatística foi feita pela aplicação do teste exato de Fisher.

\section{RESULTADOS}

A análise dos parâmetros funcionais avaliados pela ecocardiografia transepicárdica mostrou que os 14 animais apresentavam valores normais nas medidas tomadas antes da instalação da circulação extracorpórea.

Todos os cães que não receberam cardioplegia não se recuperaram após 120 minutos de parada anóxica, apresentando quadro tipo "stone heart" quando do restabelecimento da circulação coronariana, vindo a falecer.

Os 9 animais que receberam proteção miocárdica recuperaram prontamente os batimentos cardíacos quando da reperfusão com condições hemodinâmicas satisfatórias, o que possibilitou a interrupção da perfusão sem problemas. Nesse grupo de 9 animais foram realizadas novas medidas da fração de ejeção e delta $D$, respectivamente 1 e 5 horas após a suspensão da perfusão extracorpórea.
Nas medidas tomadas 60 minutos após a reperfusão, verificamos que a fração de ejeção (FE 2) apresentava alterações moderadas em 5 animais, sendo que 1 cão apresentava FE2 normal. Um animal apresentava alterações severas e em 2 cães encontrou-se discreta alteração da fração de ejeção.

Em relação às medidas de delta $D, 1$ cão apresentava alteração severa, 2 cães apresentavam alterações discretas. Esses 3 animais são os mesmos que apresentavam frações de ejeção com alterações severa e discreta. Em um dos 5 animais com frações de ejeção moderadamente alteradas, verificou-se ser o valor de delta D normal. Nos 4 restantes, havia também alterações moderadas de delta $D$

As medidas feitas 5 horas após o reinício da perfusão miocárdica mostraram que 7 cães readquiriram valores normais para a fração de ejeção e delta $D$ (Tabela 2).

Foi realizada uma tabela de freqüências cruzadas (Tabela 3) a qual mostrou que $66,67 \%$ dos cães encontravam-se com alterações moderadas e/ou severas na segunda avaliação. Concomitantemente, $33,33 \%$ dos cães encontravam-se nas categorias normal ou discreta; $55,56 \%$ dos animais apresentavam alterações moderadas e/ou severas de delta $D$ no mesmo momento, sendo que $44,44 \%$ apresentavam valores normais ou discretamente alterados.

Conforme se vê na Tabela 3, 7 animais readquirem performance normal na terceira avaliação, sendo que apenas 2 mantiveram alterações discretas. Assinale-se que esses 2 últimos animais apresentaram valores moderadamente alterados na segunda avaliação, tendo tido melhora funcional na última avaliação, quando melhoraram para discretamente alterados.

TABELA 2

\begin{tabular}{|c|c|c|c|c|c|c|c|}
\hline Grupo & Cão no. & FE1 & $\Delta \mathrm{D} 1 \%$ & FE2 & $\Delta \mathrm{D} 2 \%$ & FE3 & $\Delta \mathrm{D} 3 \%$ \\
\hline NP & 1 & $\mathbf{N}$ & $\mathbf{N}$ & & & & \\
\hline $\mathrm{P}$ & 2 & $\mathbf{N}$ & $\mathbf{N}$ & $\mathbf{M}$ & $\mathbf{M}$ & $\mathbf{N}$ & $\mathbf{N}$ \\
\hline$P$ & 3 & $\mathbf{N}$ & $\mathbf{N}$ & $\mathbf{N}$ & $\mathbf{N}$ & $\mathbf{N}$ & $\mathbf{N}$ \\
\hline NP & 4 & $\mathbf{N}$ & $\mathbf{N}$ & & & & \\
\hline $\mathrm{P}$ & 5 & $\mathbf{N}$ & $\mathbf{N}$ & M & M & D & D \\
\hline NP & 6 & $\mathbf{N}$ & $\mathbf{N}$ & & & & \\
\hline NP & 7 & $\mathbf{N}$ & $\mathbf{N}$ & & & & \\
\hline NP & 8 & $\mathbf{N}$ & $\mathbf{N}$ & & & & \\
\hline$P$ & 9 & $\mathbf{N}$ & $\mathbf{N}$ & $\mathbf{M}$ & M & $\mathbf{N}$ & N \\
\hline$P$ & 10 & $\mathbf{N}$ & $\mathbf{N}$ & M & $\mathbf{N}$ & D & D \\
\hline$P$ & 11 & $\mathbf{N}$ & $\mathbf{N}$ & M & M & $\mathbf{N}$ & $\mathbf{N}$ \\
\hline$P$ & 12 & $\mathbf{N}$ & $\mathbf{N}$ & $S$ & $\mathrm{~S}$ & $\mathbf{N}$ & $\mathbf{N}$ \\
\hline$P$ & 13 & $\mathbf{N}$ & $\mathbf{N}$ & D & D & $\mathbf{N}$ & N \\
\hline$P$ & 14 & $\mathbf{N}$ & $\mathbf{N}$ & D & D & $\mathbf{N}$ & $\mathbf{N}$ \\
\hline
\end{tabular}

$\mathrm{NP}=$ cães não protegidos. $\mathrm{P}=$ cães que receberam proteção miocárdica com cardioplegia. $\mathrm{FE}$ = fração de ejeção. Vê- se que todos os 14 animais tinham parâmetros iniciais normais $(N)$. $D=$ alteração discreta. $M=$ alteração moderada. $S=$ alteração severa. 
Dias A R, Gutierrez P S, Higuchi M L, Lourenção R, Miranda E, Santos B, Abduch M C D, Dias R R, Stolf N A G, Oliveira S A Estudo experimental em cães da ação protetora de solução cardioplégica de lidocaína e potássio. Rev Bras Cir Cardiovasc 2002; 17(1): 79-89.

\section{TABELA 3}

FREQÜÊNCIA CRUZADAS AVALIANDO-SE AS VARIAÇÕES DOS VALORES DA FRAÇÃO DE EJEÇÃO (FE) E DELTA D OCORRIDAS NAS MEDIDAS TOMADAS CERCA DE UMA HORA APÓS O RESTABELECIMENTO DOS BATIMENTOS CARDÍACOS NOS CÃES DO GRUPÓ 2

\begin{tabular}{ccc}
\hline FE 2 & FE3 & \\
$\%$ & N ou D & Total \\
N ou D & 3 & 3 \\
& 33.33 & 33.33 \\
M ou S & 6 & 6 \\
& 66.67 & 66.67 \\
Total & 9 & 9 \\
& 100.00 & 100.00 \\
\hline D2 & D3 & \\
$\%$ & ou D & Total \\
N ou D & 4 & 4 \\
& 44.44 & 44.44 \\
M ou S & 5 & 5 \\
& 55.56 & 55.56 \\
Total & 9 & 9 \\
& 100.00 & 100.00 \\
\hline
\end{tabular}

Observa-se que 66,67\% apresentavam alterações moderadas e/ou severas de FE nesta avaliação; $55,56 \%$ dos animais apresetavam alterações moderadas e/ou severas de delta $D$ no mesmo momento.

Do ponto de vista da avaliação ecocardiográfica, os resultados demonstram claramente um decréscimo de função na primeira hora após a reperfusão, que praticamente desaparece 5 horas após, havendo a obtenção de valores normais para a fração de ejeção e delta $D$.

Em todo o período pós circulação extracorpórea os cães foram mantidos anestesiados e em boas condições metabólicas e de ventilação.

$\mathrm{Na}$ avaliação anatomopatológica à microscopia óptica tem particular importância, devido a maior especificidade à presença de bandas de contração e à necrose de fibras homogêneas. A Tabela 4 apresenta os valores dos escores obtidos para as diferentes variáveis estudadas. A Tabela 5 mostra os valores do escores agrupados conforme exposto no método.

Verificamos que, no grupo de cães não protegidos (NP), em relação às bandas de contração, foram encontradas alterações severas em 3 cães e moderadas em 2. Já no grupo protegido, 7 animais não apresentavam essa alteração, sendo que em 2 essa alteração foi considerada discreta (Figuras 1 a 3). Em relação à necrose de fibras homogêneas, no grupo NP, 1 cão apresentava alteração severa. Dois animais apresentavam alteração discreta, sendo ausente nos 2 restantes.

No grupo protegido, ocorreram alterações discretas dessa variável em 5 animais, severa em 1 e ausente nos 3 restantes.
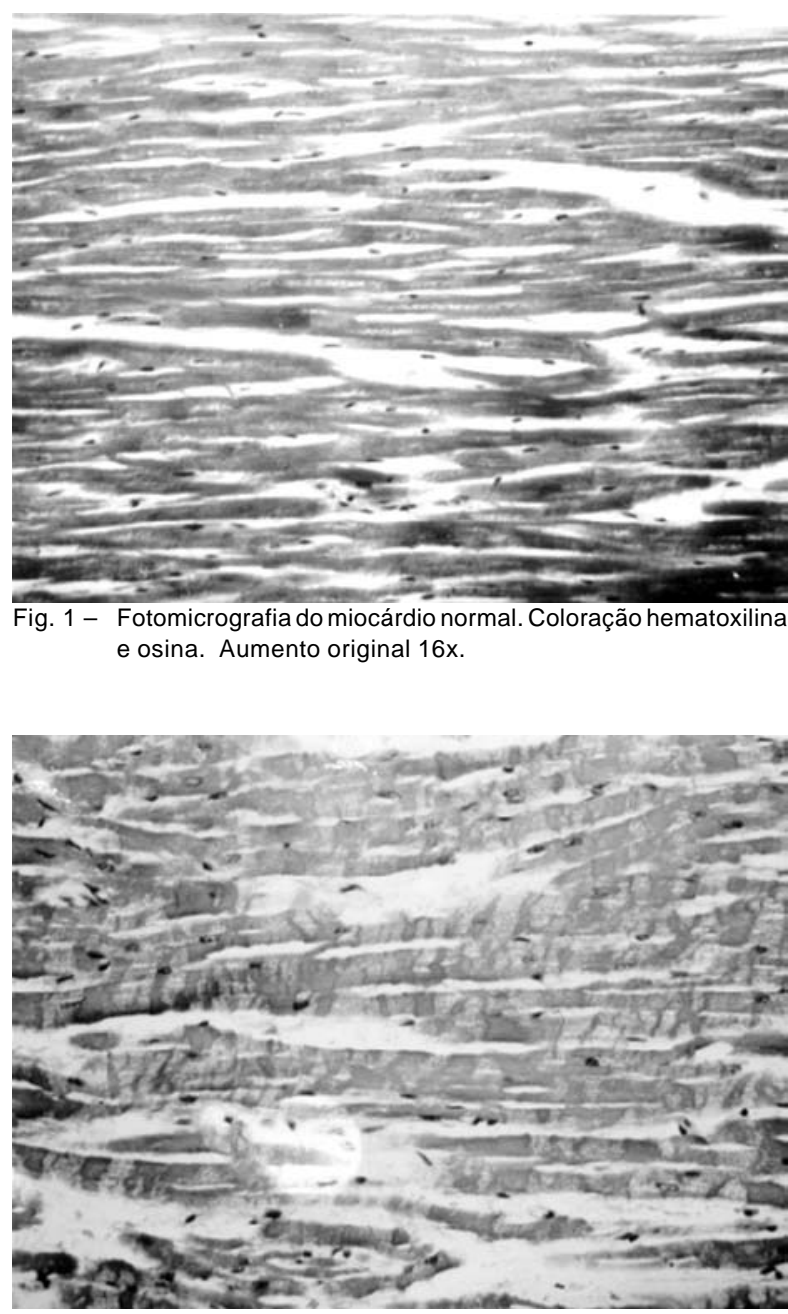

Fig. 2 - Fotomicrografia do miocárdio observando-se necrose em bandas de contração. H-E, 16x. Observa-se que as células necróticas têm faixas bem escuras, relativamente espessa, entremeadas com zonas claras, ainda mais grossas, tomando um aspecto semelhante a uma exacerbação da estriação.

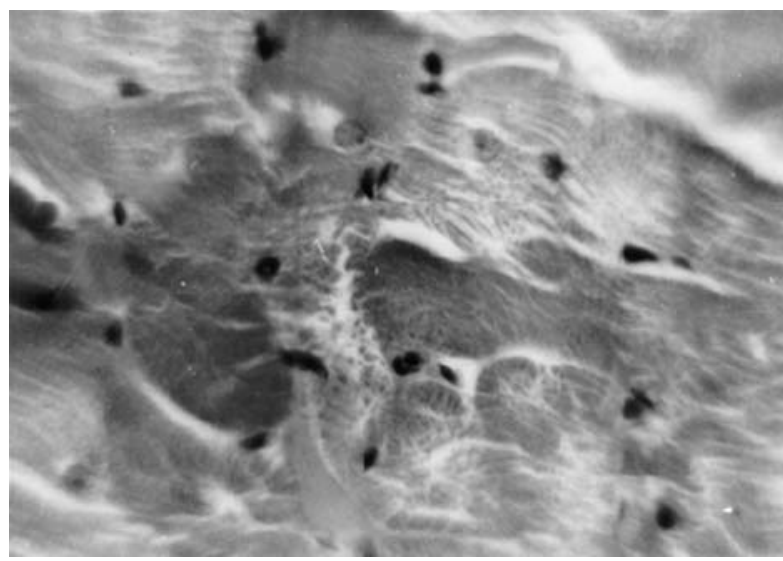

Fig. 3 - Fotomicrografia do miocárdio observando-se necrose com fibras homogêneas. Observa-se que as fibras necróticas perdem parte das estriações, ficando mais homogêneas e mais eosinofílicas (mais escuras nafoto). Coloração hematoxilinaeosina. Aumento original 40x. 
Dias A R, Gutierrez P S, Higuchi M L, Lourenção R, Miranda E, Santos B, Abduch M C D, Dias R R, Stolf N A G, Oliveira S A Estudo experimental em cães da ação protetora de solução cardioplégica de lidocaína e potássio. Rev Bras Cir Cardiovasc 2002; 17(1): 79-89.

TABELA 4

\begin{tabular}{|c|c|c|c|c|c|c|c|c|c|c|c|c|c|c|c|c|c|c|c|c|c|c|c|c|c|c|c|c|c|}
\hline \multicolumn{30}{|c|}{ VALORES OBTIDOS PARA OS ESCORES DAS VARIÁVEIS ESTUDAS "A MICROSCOPIA DE LUZ } \\
\hline \multicolumn{2}{|c|}{ Grupo Cão } & \multicolumn{3}{|c|}{ Edema } & \multicolumn{5}{|c|}{ Congestão } & \multicolumn{4}{|c|}{ Hemorragia } & \multicolumn{4}{|c|}{$\begin{array}{l}\text { Banda de } \\
\text { Contração }\end{array}$} & \multicolumn{5}{|c|}{$\begin{array}{c}\text { Necrose- } \\
\text { fibras } \\
\text { Homogêneas }\end{array}$} & \multicolumn{3}{|c|}{$\begin{array}{c}\text { Infiltração } \\
\text { por } \\
\text { neutrófilos }\end{array}$} & \multicolumn{4}{|c|}{$\begin{array}{l}\text { Onfiltração por } \\
\text { mononucleares }\end{array}$} \\
\hline & & A & $\mathrm{P}$ & VD & $\mathbf{S}$ & A & $\mathrm{P}$ & VD & $\mathbf{S}$ & A & $\mathrm{P}$ & VD & $\mathbf{S}$ & A & $\mathrm{P}$ & VD & $\mathbf{S}$ & $A$ & $P$ & VD & $\mathbf{S}$ & $A$ & $P$ & VD & $\mathbf{S}$ & $A$ & $P$ & VD & $S$ \\
\hline NP & 1 & 1 & 1 & 2 & 6 & 0 & 1 & 0 & 2 & 0 & 0 & 0 & 0 & 3 & 3 & 1 & 13 & 0 & 0 & 0 & 0 & 0 & 2 & 0 & 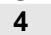 & 1 & 3 & 0 & 6 \\
\hline $\mathbf{P}$ & 2 & 2 & 1 & 1 & 7 & 0 & 0 & 0 & 0 & 0 & 0 & 0 & 0 & 0 & 0 & 0 & 0 & 1 & 0 & 1 & 3 & 0 & 0 & 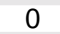 & 0 & 0 & 0 & 0 & 0 \\
\hline D & 3 & 1 & 1 & 1 & 5 & 0 & 0 & 0 & 0 & 0 & 0 & 0 & 0 & 0 & 0 & 0 & 0 & 0 & 0 & 0 & 0 & 1 & 1 & 0 & 4 & 2 & 2 & 0 & 8 \\
\hline NP & 4 & 1 & 0 & 0 & 2 & 1 & 1 & 1 & 5 & 0 & 1 & 2 & 4 & 3 & 3 & 2 & 14 & 3 & 2 & 3 & 13 & 1 & 1 & 1 & 5 & 3 & 3 & 2 & 14 \\
\hline $\mathbf{P}$ & 5 & 1 & 1 & 2 & 6 & 1 & 2 & 1 & 7 & 0 & 0 & 0 & 0 & 0 & 0 & 0 & 0 & 1 & 1 & 0 & 4 & 0 & 0 & 0 & 0 & 0 & 1 & 0 & 2 \\
\hline NP & 6 & 1 & 1 & 1 & 5 & 1 & 0 & 1 & 3 & 2 & 0 & 1 & 5 & 0 & 3 & 0 & 6 & 0 & 0 & 0 & 0 & 1 & 1 & 0 & 4 & 2 & 3 & 1 & 11 \\
\hline NP & 7 & 1 & 1 & 1 & 5 & 0 & 0 & 0 & 0 & 0 & 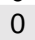 & 1 & 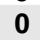 & 3 & 2 & 2 & 12 & 1 & 1 & 1 & 5 & 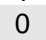 & 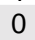 & 0 & 0 & 1 & 0 & 0 & 2 \\
\hline NP & 8 & 1 & 1 & 1 & 5 & 0 & 0 & 1 & 1 & 0 & 0 & 2 & 2 & 0 & 3 & 1 & 7 & 0 & 0 & 2 & 2 & 0 & 0 & U & 0 & 0 & 3 & 0 & 6 \\
\hline $\mathbf{P}$ & 9 & 0 & 0 & 0 & 0 & 1 & 1 & 1 & 5 & 1 & 0 & 0 & 2 & 0 & 0 & 0 & 0 & 0 & 0 & 0 & 0 & 0 & 0 & 0 & 0 & 0 & 0 & 0 & 0 \\
\hline$P$ & 10 & 1 & 0 & 1 & 3 & 0 & 1 & 1 & 3 & 0 & 0 & 0 & 0 & 0 & 0 & 0 & 0 & 0 & 0 & 2 & 2 & 0 & 0 & 2 & 2 & 0 & 0 & 2 & 2 \\
\hline $\mathbf{P}$ & 11 & 0 & 0 & 1 & 1 & 0 & 2 & 1 & 5 & 0 & 0 & 0 & 0 & 0 & 0 & 1 & 1 & 1 & 1 & 1 & 5 & 0 & 0 & 0 & 0 & 2 & 2 & 2 & 10 \\
\hline P & 12 & 0 & 0 & 0 & 0 & 1 & 0 & 0 & 2 & 2 & 0 & 0 & 4 & 1 & 0 & 0 & 2 & 2 & 2 & 2 & 10 & 0 & 0 & 0 & 0 & 3 & 3 & 2 & 14 \\
\hline $\mathbf{P}$ & 13 & 0 & 0 & 0 & 0 & 0 & 0 & 1 & 1 & 0 & 0 & 1 & 1 & 0 & 0 & 0 & 0 & 0 & 0 & 0 & 0 & 0 & 3 & 0 & 6 & 1 & 2 & 1 & 7 \\
\hline $\mathbf{P}$ & 14 & 0 & 0 & 1 & 1 & 0 & 0 & 0 & 0 & 0 & 0 & 1 & 1 & 0 & 0 & 0 & 0 & 0 & 1 & 0 & 2 & 1 & 0 & 0 & 2 & 1 & 3 & 3 & 11 \\
\hline
\end{tabular}

Nota-se que cada grandeza tem 3 valores, a saber: $A=$ intensidade de alteração ou número de cruzes no corte histológico correspondente a parede anterior do ventrículo esquerdo; $\mathrm{P}$ = intensidade de alteração em número de cruzes no corte histológico correspondente a parede posterior do VE; $\mathrm{VD}$ = intensidade de alteração ou número de cruzes no corte histológico correspondente ao ventrículo direito (VD). O número em negrito é o escore (S) obtido pela somática de: 2xA + 2xP + 1xVD; Cães protegidos pela cardiologia; NP = Cães protegidos.

TABELA 5

\begin{tabular}{|c|c|c|c|c|c|c|c|c|}
\hline \multicolumn{9}{|c|}{ ALTERAÇÕES ENCONTRADAS À MICROSCOPIA ÓPTICA } \\
\hline Grupo & $\begin{array}{c}\text { Cão } \\
\text { no }\end{array}$ & Edema & Congestão & Hemorragia & $\begin{array}{l}\text { Banda de } \\
\text { Contração }\end{array}$ & $\begin{array}{c}\text { Necrose- } \\
\text { fibras } \\
\text { Homogêneas }\end{array}$ & $\begin{array}{c}\text { Infiltração } \\
\text { por } \\
\text { neutrófilos }\end{array}$ & $\begin{array}{l}\text { Onfiltração por } \\
\text { mononucleares }\end{array}$ \\
\hline$\overline{\mathrm{NP}}$ & 1 & $\mathrm{M}$ & $\mathrm{D}$ & A & $\mathrm{S}$ & A & $\mathrm{D}$ & $\mathrm{M}$ \\
\hline $\mathrm{P}$ & 2 & M & A & A & A & $\mathrm{D}$ & A & A \\
\hline $\mathrm{P}$ & 3 & $\mathrm{D}$ & A & A & A & A & $\mathrm{D}$ & M \\
\hline NP & 4 & $D$ & $D$ & $D$ & $S$ & $S$ & $D$ & $S$ \\
\hline P & 5 & $M$ & $M$ & A & $A$ & $D$ & $A$ & $A$ \\
\hline NP & 6 & $D$ & $D$ & $\mathrm{D}$ & $\mathrm{M}$ & $A$ & $D$ & $S$ \\
\hline NP & 7 & D & A & $A$ & $S$ & $D$ & $A$ & $D$ \\
\hline NP & 8 & $D$ & D & $D$ & $M$ & $D$ & $A$ & $M$ \\
\hline$P$ & 9 & A & D & $D$ & A & A & $A$ & A \\
\hline$P$ & 10 & $D$ & $\mathrm{D}$ & $A$ & $A$ & $D$ & $D$ & $D$ \\
\hline$P$ & 11 & D & D & A & $D$ & $D$ & A & $S$ \\
\hline$P$ & 12 & A & $D$ & $D$ & $D$ & $S$ & $A$ & $S$ \\
\hline$P$ & 13 & A & D & $D$ & $A$ & A & $M$ & $M$ \\
\hline$P$ & 14 & $D$ & $A$ & $D$ & $A$ & $D$ & $D$ & $S$ \\
\hline
\end{tabular}

Valores das alterações encontradas à microcospia óptica, agrupando-se os valores dos escores das diferentes grandezas apresentados na tabela 4 , de acordo como exposto na metodologia. $A=$ ausente; $D=$ discreta; $M=$ moderada; $S=-$ severa.

O teste exato de Fisher mostrou diferença altamente significativa entre os grupos NP e $P$, no que se refere às bandas de contração $(p=0,0005)$.

As demais variáveis estudadas mostraram clara tendência a alterações mais intensas no grupo não protegido, embora não tenha havido significância estatística.

Foram vistas alterações menores em todos os casos, tais como dilatação do retículo sarcoplasmático, certo grau de tumefação mitocondrial, bem como retificação e destruição das cristas dessa organela.

Em relação aos resultados obtidos na microscopia eletrônica dentro da padronização de escores que foi seguida, verificamos que, no grupo NP, $60 \%$ dos animais apresentavam alterações graves, as quais foram encontradas em $22,22 \%$ do grupo protegido (Tabela 1). 
Dias A R, Gutierrez P S, Higuchi M L, Lourenção R, Miranda E, Santos B, Abduch M C D, Dias R R, Stolf N A G, Oliveira S A Estudo experimental em cães da ação protetora de solução cardioplégica de lidocaína e potássio. Rev Bras Cir Cardiovasc 2002; 17(1): 79-89.

Alterações moderadas foram encontradas em $40 \%$ do grupo não protegido e em $33,33 \%$ do grupo protegido.

Já no grupo protegido pela cardioplegia, 44,44\% apresentavam alterações consideradas leves.

Estes resultados sinalizam fortemente para maior severidade de lesões ultramicroscópicas no grupo não protegido (Figuras 4 e 5 e Tabela 1).

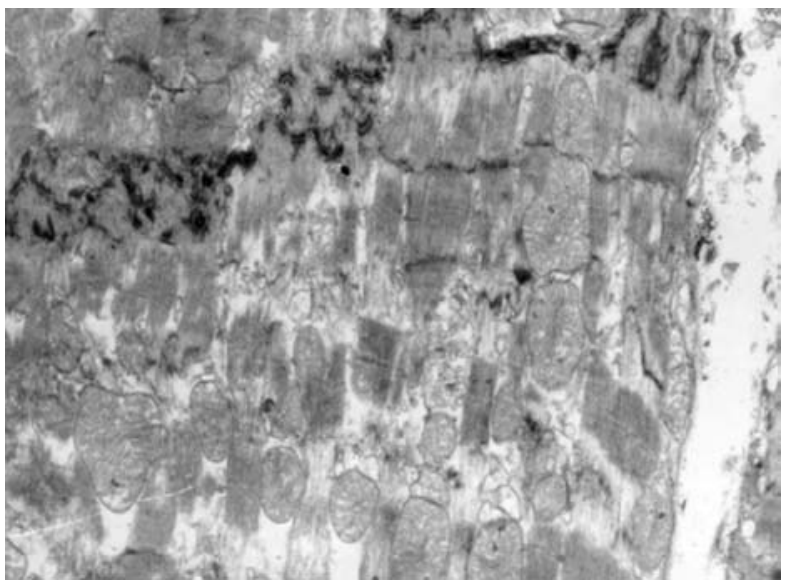

Fig. 4 - Ultramicrografia de miocárdio necrótico. Aumento original 2000x. Observa-se área com bandas de contração. As miofilamentos tem extensas faixas claras entremeadas com zonas em que os microfilamentos estão agrupados. Comparar com as miofibrilas ao alto com aspecto normal.

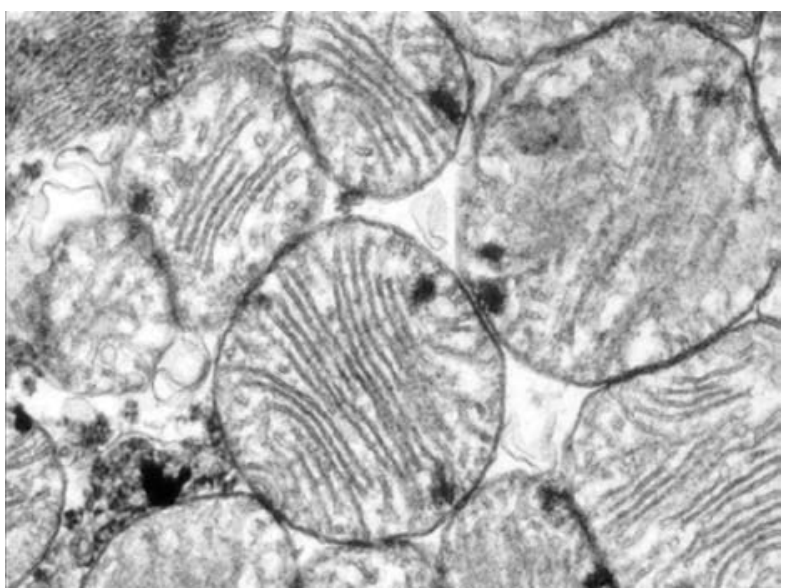

Fig. 5 - Ultramicrografia mostrando mitocondrias com densidade amorfas (grumos endurecidos). Há também perdas das cristas em algumas delas. Aumento original 1000x.

\section{COMENTÁRIOS}

Corações de cães, quando operados com perfusão extracorpórea e submetidos a parada cardíaca anóxica por pinçamento transversal da aorta ascendente, apresentam repercussões funcionais e anatomopatológicas quando da reperfusão, de intensidades variáveis e proporcionais ao tempo de isquemia.
DALLAN ${ }^{(24)}$, em nosso meio, estudou o assunto em tese de doutoramento apresentada à FMUSP, em 1986. Demonstrou que cães submetidos a 60 minutos de parada cardíaca e reperfundidos posteriormente apresentavam intensas repercussões funcionais, com significativa queda da pressão arterial, elevação da pressão capilar pulmonar e acentuada queda do débito cardíaco. Nesses animais, os estudos anatomopatológicos realizados também demonstravam acentuadas alterações representativas de graus variáveis de necrose miocárdica.

Neste trabalho, demonstramos que, no grupo controle submetido a parada cardíaca por $120 \mathrm{mi}-$ nutos, os efeitos deletérios da isquemia foram tão intensos que nenhum cão sobreviveu. Os estudos anatomopatológicos revelaram alterações intensas compatíveis com necrose miocárdica.

Os animais protegidos com a técnica de cardioplegia aqui apresentada mostraram diferenças marcantes com o grupo controle. Assim é que, em primeiro lugar, não houve óbitos em nenhum desses cães. Em segundo lugar, a função cardíaca apresentou uma redução de cerca de $30 \%$ após a primeira hora de reperfusão, vindo a normalizar-se cerca de 5 horas após a reinstalação da circulação coronariana.

Estes fatos marcantes foram obtidos à custa da ação protetora da lidocaína, a qual já havia sido demonstrada por vários trabalhos $(4,7)$.

ZHOU et al. (8) estudaram a ação protetora da lidocaína sobre o sistema nervoso central em cães submetidos a parada circulatória total, submetidos a perfusão extracorpórea com canulação pela artéria femoral direita e drenagem venosa pela veia jugular externa direita. Mantiveram o tórax fechado e utilizaram resfriamento de superfície para baixar a temperatura a $20^{\circ} \mathrm{C}$. A essa temperatura, realizaram parada circulatória total por 90 minutos. Os animais foram divididos em 2 grupos: grupo controle e grupo no qual injetaram lidocaína na dose de $4 \mathrm{mg} /$ $\mathrm{kg}$ cerca de 2 minutos antes da parada circulatória e $2 \mathrm{mg} / \mathrm{kg}$ por ocasião do restabelecimento da circulação e do reaquecimento. As avaliações neurológicas realizadas no pós-operatório revelaram desempenho muito melhor no grupo em que se associou a lidocaína. Os animais foram sacrificados após 8 dias, sendo que os estudos anatomopatológicos revelaram alterações muito mais intensas nos cães não protegidos pela lidocaína. Os autores não referem óbitos nos 2 grupos, o que nos leva a inferir que o coração tolerou bem 90 minutos de parada circulatória a $20^{\circ} \mathrm{C}$, diferentemente do que ocorre em condições de normotermia, como já demonstrado por DALLAN ${ }^{(24)}$ e neste trabalho, com 60 e 120 minutos de anóxia, respectivamente. 
Dias A R, Gutierrez P S, Higuchi M L, Lourenção R, Miranda E, Santos B, Abduch M C D, Dias R R, Stolf N A G, Oliveira S A Estudo experimental em cães da ação protetora de solução cardioplégica de lidocaína e potássio. Rev Bras Cir Cardiovasc 2002; 17(1): 79-89.

Outro dado interessante são as dosagens de lidocaína utilizada. Para um cão de $25 \mathrm{~kg}$, peso médio da maioria dos cães usados neste experimento, equivaleria a dizer uma infusão de $150 \mathrm{mg}$ de lidocaína em 90 minutos. Neste trabalho, utilizamos apenas cerca de $4 \mathrm{mg} / \mathrm{kg}$ de peso como dose única.

SUNAMORI et al. (9) estudaram experimentalmente em cães as ações de diferentes soluções cardioplégicas: magnésio e lidocaína, potássio e magnésio e apenas potássio. Usaram soluções cristalóides e perfusão extracorpórea com hipotermia a $20^{\circ} \mathrm{C}$. Submeteram os animais a períodos de isquemia de 60 e 120 minutos, tendo dois grupos controles e um terceiro grupo no qual realizaram apenas toracotomia.

Analogamente ao que ocorreu no trabalho de ZHOU et al. ${ }^{(8)}$, os cães submetidos a períodos de anóxia de 60 e 120 minutos sobreviveram. Entretanto, os estudos de função mitocondrial relatados demonstram lesões nas isquemias mais prolongadas. Os grupos que utilizaram soluções cardioplégicas e foram submetidos a isquemias durante 60 minutos não apresentaram grandes diferenças quanto aos parâmetros estudados. Entretanto, com 120 minutos de isquemia, a solução com magnésio e lidocaína apresentou um efeito protetor bastante significativo quando comparada às demais. Os autores chamam a atenção para o fato de que a hipotermia a $20^{\circ} \mathrm{C}$, por si só, protege efetivamente o miocárdio quando a isquemia não ultrapassa 60 minutos. Embora feito com hipotermia acentuada, os resultados aqui obtidos confirmam os de DALLAN (24) e guardam alguma relação com os resultados de nosso trabalho.

SUNAMORI et al. (11) estudaram, em 1982, comparativamente, os efeitos protetores de soluções cardioplégicas, em cães submetidos a parada cardíaca por períodos de 120 minutos. Analisaram solução com potássio, insulina e glicose e solução com magnésio e lidocaína. Avaliaram a função cardíaca após 60 minutos de perfusão, bem como a ultraestrutura do miocárdio.

Demonstraram que, no grupo que foi protegido com solução cardioplégica à base de magnésio e lidocaína, o desempenho funcional do ventrículo esquerdo foi praticamente normal aos 60 minutos de reperfusão. Entretanto, após 120 minutos de isquemia sob hipotermia, o efeito protetor da cardioplegia com lidocaína foi evidente. No nosso material, verificamos que, na primeira hora após a reperfusão, houve uma queda de cerca de $30 \%$ na função ventricular, a qual retorna aos valores normais iniciais após 5 horas. Tal diferença, talvez possa ser explicada pela diferença de metodologia utilizada.

Os estudos bioquímicos e ultramicroscópicos do trabalho de SUNAMORI et al. ${ }^{(11)}$ mostram também a superioridade da solução de magnésio e lidocaína.

Os autores chamam a atenção para as ações da lidocaína. Essa substância inibe o efluxo de potássio das mitocondrias e inibe o influxo de sódio, com efeito discreto sobre afluxo de cálcio através das membranas do sarcolema durante os potenciais de ação.

KYO et al. (12) estudaram também o uso de soluções cardioplégicas com lidocaína normocalêmicas e hipercalêmicas. Utilizaram cães nos quais foram injetadas as soluções sob hipotermia de $28^{\circ} \mathrm{C}$ e fizeram estudos hemodinâmicos, enzimáticos e morfológicos. Constataram que, no grupo em que se usou cardioplegia com lidocaína e baixas concentrações de potássio, os resultados foram significativamente superiores.

Em nosso material, os $100 \mathrm{mg}$ de lidocaína foram associados a $2,5 \mathrm{~m} / \mathrm{Eq}$ de potássio. As demais infusões feitas a cada 20 minutos veicularam apenas sangue oxigenado sob pressão sistêmica. Essa baixa concentração de potássio injetada na primeira infusão talvez tenha colaborado com os bons resultados obtidos. Não houve, em nenhum momento de todos os experimentos, variação significativa das concentrações de potássio sangüíneo, as quais foram mantidas no limite superior da normalidade. As reinfusões, ao se veicular sangue da linha arterial, contribuíram para o que se chama na literatura de parada aeróbica.

Pelo exposto, entendemos que o método de cardioplegia utilizado foi eficiente na proteção do miocárdio.

\section{CONCLUSÕES}

a) Cães colocados em perfusão normotérmica e submetidos a parada cardíaca anóxica por 120 minutos, quando reperfundidos, apresentam o quadro de "stone heart", não recuperando os batimentos. Os estudos anatomopatológicos à microscopia de luz e à ultramicroscopia revelam danos celulares intensos, compatíveis com necrose celular.

b) O uso de cardioplegia sangüínea normotérmica, fazendo-se a indução com a infusão de $100 \mathrm{mg}$ de lidocaína e 2,5 $\mathrm{mEq}$ de potássio e sangue arterial, provoca parada cardíaca imediata. 
Dias A R, Gutierrez P S, Higuchi M L, Lourenção R, Miranda E, Santos B, Abduch M C D, Dias R R, Stolf N A G, Oliveira S A Estudo experimental em cães da ação protetora de solução cardioplégica de lidocaína e potássio. Rev Bras Cir Cardiovasc 2002; 17(1): 79-89.

c) A manutenção da parada cardíaca é obtida com injeções repetidas a cada 20 minutos de sangue proveniente da linha arterial do circuito de perfusão, durante 30 segundos.

d) Verificamos queda da função contrátil de cerca de $30 \%$ na primeira hora após a reperfusão coronariana.

e) Todos os animais apresentaram função contrátil normal, quando estudados novamente cerca de 5 horas após a reperfusão. f) No grupo de cães protegidos pelo método de cardioplegia em estudo, as alterações anatomopatológicas, a microscopia óptica e ultramicroscopia, encontradas após o sacrifício dos animais, foram inexpressivas.

g) A cardioplegia proposta é um método eficiente de proteção miocárdica, tanto do ponto de vista funcional como sob a análise morfológica.

RBCCV 44205-575

Dias A R, Gutierrez P S, Higuchi M L, Lourenção R, Miranda E, Santos B, Abduch M C D, Dias R R, Stolf N A G, Oliveira S A - Experimental study of the protection provided by a cardioplegic solution of lidocaine and potassium in dogs. Rev Bras Cir Cardiovasc 2002; 17(1): 79-89.

ABSTRACT: Introduction: The action of a cardioplegic solutions with lidocaine and potassium. Was studied experimentally in dogs.

Material and Methods: Fourteen dogs, divided en two groups, were operated.

The group 1 was composed of 5 dogs, who were submitted to 120 minutes of myocardial anoxia, through cross clamping of the ascending aorta under cardiopulmonary bypass, without any myocardial protection. The group 2 were treated in the same way, but received cardioplegic protection. The technique of cardioplegia consisted of two phases: a) Induction phase the induction was achieved by the injection of $100 \mathrm{mg}$ of lidocain and $2.5 \mathrm{mEq}$ of potassium, diluted in $60 \mathrm{ml}$ of blood from the arterial line. This first injection was in bolus. After this, blood was injected from the arterial line during 30 seconds. The heart immediately had an arrest. b)The second phase was called maintaining phase. This phase consisted of repeated injections of blood from the arterial line, during 30 seconds each, and repeated every 20 minutes during the ischemic period.

Results: All animals of this protected group survived after the release of the cross clamping of the aorta and all the dogs of the non protected group died after the end of the ischemic time. Echocardiographic evaluation were performed in 3 different moments of the experiment. The first was control, before cardiopulmonary bypass and the measures of delta $D$ and ejection fraction were normal in all animals. The second was done about one hour after the reperfusion, in the group 2 animals. This measurement showed a decrease of $30 \%$ when compared with the control values. The third echocardiographic evaluation was done 5 hours after the end of the perfusion and showed a restoring to normal values in all 9 dogs of the group 2 dogs. All 14 hearts were studied with optical and electronic morphological exams. The group 1 dogs showed intense myocardial damage, while the group 2 animals showed inexpressive morphological findings.

Conclusion: It was concluded that the cardioplegia technique utilized in group 2 animals was effective in myocardial protection action, with good echocardiographic performance after perfusion and almost no morphological alterations in anatomopathological studies.

DESCRIPTORS: Cardioplegic sulutions. Lidocaine, therapeutic use. Potassium, use. 
Dias A R, Gutierrez P S, Higuchi M L, Lourenção R, Miranda E, Santos B, Abduch M C D, Dias R R, Stolf N A G, Oliveira S A Estudo experimental em cães da ação protetora de solução cardioplégica de lidocaína e potássio. Rev Bras Cir Cardiovasc 2002; 17(1): 79-89.

\section{REFERÊNCIAS BIBLIOGRÁFICAS}

1 Gianelly R, von der Groeben J O, Spivack A P, Harrison D C - Effect of lidocaine on ventricular arrhythmias in patients with coronary heart disease. $N$ Engl $J$ Med 1967; 277: 1215-9.

2 Bigger J R \& Hoffman B F - Drogas antiarrítmicas. In: Goodman \& Gilman, eds. As bases farmacológicas da terapêutica. 8. Ed. Rio de Janeiro: Editora Guanabara Koogan, 1991; 554-74.

3 Neme B - Obstetrícia básica. São Paulo: Sarvier, 1995; $262-4$.

4 Okamura T, Sunamori M, Suzuki A - Protective effect of lidocaine in reperfused ischemic myocardium: evaluation by hemodynamic and biochemical study. Jpn Circ J 1982; 46: 657-62.

5 Sunamori M, Okamura T, Amano J, Suma H, Suzuki A - Myocardial protection by lidocaine hydrochloride in aorto - coronary bypass surgery. Jpn J Surg 1982; 12(2): 93-7.

6 Sultan I, Sunamori M, Suzuki A - Heart preservation: analysis of cardioprotective infusate characteristics: membrane stabilization, calcium antagonism, and protease inhibition on myocardial viability: a biochemical, ultrastructural, functional study. J Heart Lung Transplant 1992; 11: 607-18.

7 Lee R, Nitta T, Schmid R A, Schuessler R B, Harris KM, Gay W A Jr - Retrograde infusion of lidocaine or Larginine before reperfusion reduces myocardial infarct size. Ann Thorac Surg 1998; 65:1353-9.

8 Zhou Y, Wang D, Du M et al - Lidocaine prolongs the safe duration of circulatory arrest during deep hypothermia in dogs. Can J Anaesth 1998; 45: 692-8.

9 Sunamori M \& Harrison C E Jr - Myocardial respiration and edema following hypothermic cardioplegia and anoxic arrest. J Thorac Cardiovasc Surg 1979; 72: 208-16.

10 Hearse D J, O'Brien K, Braimbridge M - Protection of the myocardium during ischemic arrest: doseresponse curves for procaine and lignocaine in cardioplegic solutions. J Thorac Cardiovasc Surg 1981; 81: 873-9.

11 Sunamori M, Amano J, Okamura T, Suzuki A - Superior action of magnesium - lidocaine -1-aspartate cardioplegia to glucose-insulin-potassium cardioplegia in experimental myocardial protection. Jpn $J$ Surg 1982; 12: 372-80.
12 Kyo S, Laraia P J, Magrassi $\mathrm{P}$ et al. - Myocardial protection by lidocaine during cardioplegia. J Surg Res 1983; 34: 533-42.

13 Leicher F G, Magrassi P, Laraia P J, Derkac W M, Buckley M J, Austen G - Blood cardioplegia delivery: deleterious effects of potassium versus lidocaine. Ann Surg 1983; 198: 266-72.

14 Bengtsson L, Gunnes S, Norman B, Karlsson J - The effect of lidocaine on myocardial ischemia with asanguinous reperfusion: an in vitro study. Scand $J$ Thorac Cardiovasc Surg 1991; 25:141-6.

15 Baraka A, Hirt N, Dabbous A et al. - Lidocaine cardioplegia for prevention of reperfusion ventricular fibrillation. Ann Thorac Surg 1993; 55: 1529-33.

16 Wallace S R \& Baker A B - Incidence of ventricular fibrillation after aortic cross-clamp release using lignocaine cardioplegia. Anaesth Intensive Care 1994; 22: $442-6$.

17 Fiore A C, Naunheim K S, Taub J et al. - Myocardial preservation using lidocaine blood cardioplegia. Ann Thorac Surg 1990; 50: 771-5.

18 Lichtenstein S V, Ashe K A, el Dalatil H, Custimano R J, Panos A, Slutsky A S - Warm heart surgery. J Thorac Cardiovasc Surg 1991; 101: 269-74.

19 Demmy T L, Haggerty S P, Boley T M, Curtis J J - Lack of cardioplegia uniformity in clinical myocardial preservation. Ann Thorac Surg 1994; 57: 648-51.

20 Miranda E, Hernandez G, Coloma R et al. - Sistema simplificado de cardioplegia sangüínea oxigenada normotérmica. $10^{\circ}$ Congresso Brasileiro de Circulação Extracorpórea. São Paulo, 11 e 12 de setembro, 1992. Resumos.

21 Weyman A E - Principles and practice of echocardiography. 2. ed. Philadelphia: Lea \& Febiger, 1994.

22 Bonagura J D - Quantitative cross in the normal dog. Vet Radiol 1986; 27: 34-49.

23 Bobinec G L - Échocardiographie temps- mouvement: principes de base et intéret chez le chien. Pratique Médicale et Chirurgicale de l'Anim de Cie 1988; 23: 27-34.

24 Dallan L A O - Proteção miocárdica por hipotermia e cardioplegia. Aspectos enzimáticos, hemodinâmicos e ultra-estruturais: estudo experimental. [Tese de Doutorado] Faculdade de Medicina da Universidade de São Paulo. São Paulo, 1986. 